\title{
LAS MONEDAS DE SALVACAÑETE (CUENCA) Y SU SIGNIFICADO EN EL TESORO
}

\author{
POR \\ CRUCES BLÁZQUEZ y M. ${ }^{a}$ PAZ GARCÍA-BELLIDO \\ Universidad de Salamanca y CSIC, Madrid
}

\section{RESUMEN}

Las monedas del Tesoro de Salvacañete (Cuenca) del s. I a.C., fueron publicadas en 1936 sin ilustraciones por lo que existen datos muy discordantes. Hasta hoy se habían identificado las piezas del Museo Arqueológico Nacional y las del Museo de Cuenca. Añadimos aquí por primera vez los posibles ejemplares depositados por Gómez Moreno en el Instituto Valencia de Don Juan de Madrid y proponemos una reconstrucción de este material numismático. El estudio monetario parece indicar una clara selección de las monedas por su iconografía con un objetivo cultual en el que el caballo y el toro tendrían especial significado, obviando otros tipos monetales que por cronología deberían estar presentes. Podría tratarse de exvotos a una divinidad relacionada con los animales, quizás semejante a una Artemis a juzgar por la abeja representada en uno de los objetos del Tesoro.

\section{SUMMARY}

The absence of coin-illustrations from Cabre's 1936 publication of the 1 st century BC assemblage from Salvacañete (Cuenca) has been a source of confusion. Previous accounts of the coin-element have usually been limited to the pieces housed either in the Museo Arqueológico Nacional, Madrid, or in the Museo de Cuenca. The present revision takes account additionally of specimens deposited by Gómez-Moreno in the Instituto Valencia de Don Juan, Madrid, which almost certainly form part of the original find. The numismatic material appears to have been deliberately chosen for its iconographic content, with issues featuring either horses or bulls being preferred to contemporary coins of other types. A possible explanation is that they represent offerings to a divinity with animal associations; the depiction of a bee on one of the assemblage's non-numismatic items would suggest a cult similar to that of Artemis.

\section{CIRCUNSTANCIAS DEL HALLAZGO}

En 1934 se detectó en el comercio de antigüedades de Madrid la presencia de un tesorillo hallado, al parecer por un cazador, en el término municipal de Salvacañete (Cuenca) ${ }^{1}$. Se trataba de un impor-

1 Además, junto a la ermita del pueblo se ha documentado un asentamiento rural romano fechado en el s. IV, pero no contamos con otros testimonios que certifiquen la existencia de una secuencia cultural continuada. Cf. TIR (K-30), 196. Las abreviaturas utilizadas en el catálogo son las siguientes: $\mathrm{CNH}=$ Villaronga, L. Corpus Nummum Hispaniae ante ae tatis Augustum, Madrid 1994; G-B \& B = García-Bellido, M.P. \& Blázquez, C.: Las monedas celtibéricas y sus contramarcas en el Instituto Valencia de Don Juan, AN 17-18, tante conjunto de piezas de plata de ajuar doméstico y aderezo personal acompañadas por denarios ibéricos y romano-republicanos. Por mediación de M. Gómez Moreno fué adquirido por el Estado español y, tras un período de depósito en el Instituto Valencia de Don Juan, mientras se realizaban las gestiones oportunas, ingresó definitivamente en el Museo Arqueológico Nacional en 1941. Sabemos además que el MAN adquirió en 1954 un nuevo lote de joyas y monedas de la misma procedencia y que, posteriormente, familiares del descubridor del tesoro, donaron al Museo de Cuenca nuevas piezas. Sin embargo, al realizar el recuento de las monedas descritas en las distintas publicaciones, se podía observar que la cifra total variaba de unos autores a otros y que el conjunto numismático dado a conocer hasta la fecha estaba incompleto ${ }^{2}$.

\section{ESTADO DE LA CUESTIÓN}

La ausencia total de ilustraciones en la publicación original y la dispersión de las monedas han sido las principales causas de la imposibilidad de un estudio completo. La primera noticia del hallazgo la proporciona J. Cabré, quien describe de forma pormenorizada los vasos y joyas aparecidos en Salvacañete, adjuntando fotografías de dicho material; sin

1988, 59-87; Misc. = Gómez Moreno, M.: Misceláneas de Arte y Arqueología, Madrid 1949; Notas...= Gómez Moreno, M.: Notas sobre numismática hispana, Anuario del Cuerpo de Archiveros, Bibliotecarios y Arqueólogos 2, 1934, lám. 4, $\mathrm{n}^{\circ} 12-14$ y 17; RRC = Crawford, M.: Roman Republican Coinage, Cambridge 1974, 2 vols.; Vill. = Villaronga, L.: Las monedas de Arse-Saguntum, Barcelona 1967; Id., Les monedes ibèriques de Tàrraco, Tarragona 1983; Vives= Vives, A.: La moneda hispánica, Madrid 1926, 2 vols.

2 Debemos a A. Arévalo la noticia del proyecto sobre «Cambio económico y cambio ideológico. El concepto de valor a través de los depósitos ibéricos de plata con monedas» cuyo investigador principal es A. Perea. Una presentación de las monedas identificadas en el MAN oímos a A. Arévalo en Berlín, XI Congreso Internacional de Numismática, septiembre 1997. Ello nos ha animado a presentar aquí las piezas inéditas guardadas en el IVDJ, con un comentario histórico que se adecúa bien, parece ser, a la interpretación propuesta por A. Arévalo. 
embargo, las monedas no corrieron la misma suerte y el propio Cabré dió únicamente una breve descripción acompañada por la referencia a la obra de A. Vives ${ }^{3}$. Esta labor de catalogación fué llevada a cabo por M. Gómez Moreno, autor que unos pocos años después vuelve a hacer una breve relación del tesoro ${ }^{4}$.

De nuevo, en 1947, J. Cabré se refiere al conjunto de Salvacañete informándonos de que ha sido adquirido por el MAN ${ }^{5}$. Unos años después F. Alvarez Ossorio incide en la misma línea, aunque en su publicación no se hace referencia alguna a las monedas ${ }^{6}$. Posteriormente, a través de A. Fernández Avilés tenemos noticia de un nuevo ingreso en el Museo de un pequeño lote, de joyas y denarios, desgajado del conjunto inicial ${ }^{7}$.

En 1969, K. Raddatz nos ofrece un nuevo inventario del tesoro pero su información se basa en los anteriores trabajos y por tanto no aporta ningún dato nuevo ${ }^{8}$. Habrá que esperar hasta 1971 en que J.M. Navascués publica de forma detallada, y con sus correspondientes fotografías, las monedas procedentes del tesoro de Salvacañete depositadas en el $\mathrm{MAN}^{9}$. Él es el primero en observar las contradicciones existentes respecto a la cifra total de monedas entre las anteriores notificaciones. Sobre este punto, fundamental para la reconstrucción del tesoro, volveremos infra.

Finalmente, en 1976, M. Osuna da a conocer un tercer y último lote de joyas y denarios, donado al Museo de Cuenca por familiares del descubridor del tesoro, que inicialmente pudo haber formado parte del mismo ${ }^{10}$.

En resumen, el Museo Arqueológico Nacional compró en diferentes años un total de sesenta y ocho monedas del tesoro de Salvacañete: en 1941 ingresaron en el MAN cuarenta y nueve denarios de bolśkan, cinco de ikalesken y doce romanos, es decir un total de sesenta y seis monedas y en 1954 el

${ }^{3}$ Cabré Aguiló, J.: El tesoro de plata de Salvacañete (Cuenca), AEspA, 34, 1936, 151-159.

${ }^{4}$ Gómez Moreno, M.: Misc. 182.

5 Cabré Aguiló, J.: Objetos de plata del s. I, hallados en Salvacañete (Cuenca), Adquisiciones del MAN (1940-1945), Madrid, 1947, 59-62.

${ }^{6}$ Alvarez Ossorio, F.: Tesoros españoles antiguos en el Museo Arqueológico Nacional, BRAH 135, 1954, 298-301.

7 Fernández Avilés, A.: Nuevas piezas de plata del tesoro ibérico de Salvacañete (Cuenca), MMAP (1954), 15, 1958, 35-38.

${ }^{8}$ Raddatz, K.: Die Schatzfunde der Iberischen Halbinsel, Berlín, 1969, 244-249.

9 Navascués, J.M.: Las monedas hispánicas del Museo Arqueológico Nacional de Madrid, t. 2, Barcelona, 1971, 38 y $57-58$.

${ }^{10}$ Osuna Ruiz, M.: Nuevas piezas del tesoro de Salvacañete en el Museo de Cuenca, RABM 79, 1976, 389-395.
Museo adquirió otros dos denarios de bolśkan de la misma procedencia. Sin embargo, la cifra total de ejemplares hallados varía según los autores: según Cabré serían setenta y cuatro, aunque de su inventario resultan setenta y cinco, según Raddatz setenta y seis y según Gómez Moreno eran «más de setenta y siete». Esta última información, aunque imprecisa, parece más exacta.

\begin{tabular}{|l|c|c|c|}
\hline & Cabré & G. Moreno & Raddatz \\
\hline arse & 2 & 2 & 2 \\
ikalesken & 8 & 10 & 8 \\
iltirtaśalirban & 1 & 1 & 1 \\
bolśkan & 50 & más de 50 & 52 \\
kese & 1 & 1 & 1 \\
sekaisa & 1 & 1 & 1 \\
romano-repub. & 12 & 12 & 11 \\
\hline Total & 75 & más de 77 & 76 \\
\hline
\end{tabular}

Fig. 1.-Información sobre el contenido numismático del Tesoro de Salvacañete.

Como comentamos al principio, M. Gómez Moreno depositó el tesoro de Salvacañete, durante algún tiempo, en el Instituto Valencia de D. Juan y ello nos hizo pensar que quizás algunos de los ejemplares cuyo paradero se desconocía pudo haber sido adquirido por el IVDJ o, incluso, por el propio don Manuel a título personal. La identificación de las piezas del tesoro guardadas en el Instituto se ha hecho gracias a la catalogación que hemos efectuado de sus monedas antiguas y a la existencia de un álbum con las improntas de las colecciones Buckler, Sánchez de la Cotera ${ }^{11}$, Jordana y Gómez Moreno que nos ha permitido ir excluyendo las piezas que ya pertenecían al Instituto antes de la muerte de Vives y que por tanto no pudieron formar parte del tesoro de Salvacañete puesto que su hallazgo es posterior.

\section{CATÁLOGO DE LAS PIEZAS CONSERVA- DAS EN EL IVDJ}

- Monedas de arse : según la descripción de J. Cabré y M. Gómez Moreno se trata de dos piezas taladradas que responden a la clasificación de Vives lám. $6 \mathrm{n}^{\circ} 4 \mathrm{y} \mathrm{n}^{\circ} 12$. Efectivamente en el IVDJ hay dos ejemplares que responden a estas características y que además aparecen en el álbum de improntas de la colección Gómez Moreno, que en parte pasó al IVDJ, por lo que creemos que debe tratarse de las monedas de Salvacañete.

\footnotetext{
11 García-Bellido, M.P. y García de Figuerola, M., Álbum de moneda íbero-romana de la antigua colección Sánchez de la Cotera, Madrid, 1987.
} 


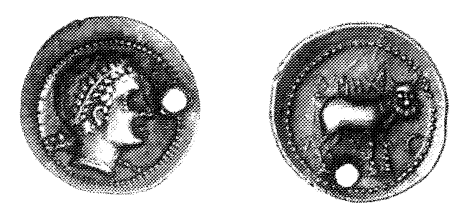

2, a
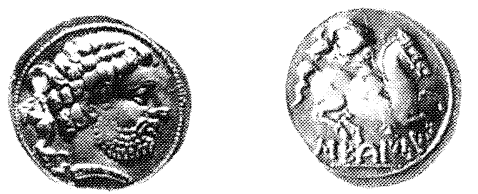

$2, \mathrm{c}$
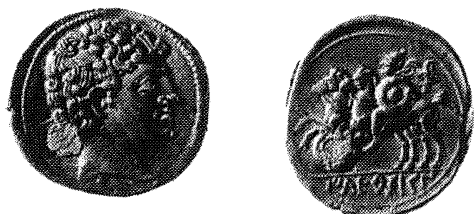

2, e
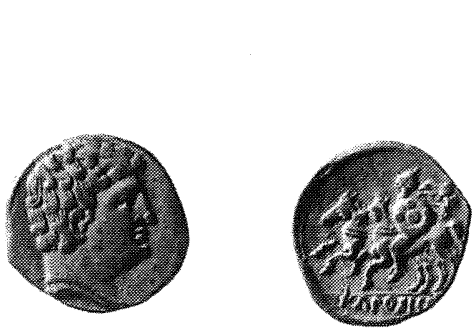

$2, \mathrm{~d}$
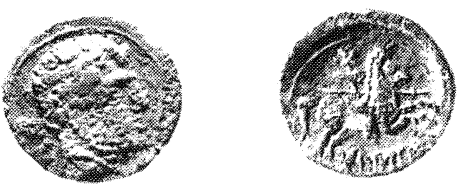

2, f

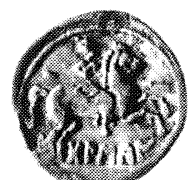

$2, \mathrm{~g}$
$2, \mathrm{~b}$

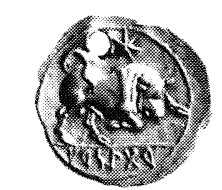

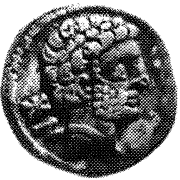
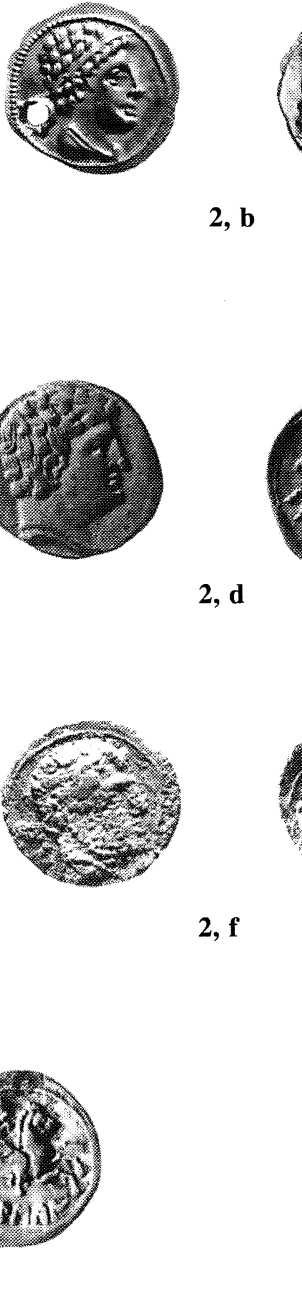



Fig. 2.-Monedas identificadas como del Tesoro de Salvacañete en el Instituto Valencia de Don Juan (a-b de arse; c de śekaisa; d-e de ikalesken; f-g de bolśkan). Esc. 1:1.

- Fig. 2, a) Ag. 2'80 g , eje 9. Vives 6, 4 ; Vill. clase IV tipo I n ${ }^{\circ} 37$; $\mathrm{CNH} 307,17$.

- Fig. 2, b) Ag. 2'82 g , eje 8. Vives 6, 12; Vill. clase VII tipo I grupo II; CNH 308, 26.

- Denario de śekaisa : es quizás la pieza más conocida del tesoro de Salvacañete por tratarse de un unicum, ya ilustrado por M. Gómez Moreno en las Misceláneas y en cuyo álbum de improntas figura. Este único denario confirma que Segeda acuñó plata muy tempranamente, ya desde sus primeras emisiones, las de cabeza barbada con leona detrás, emisión hallada en Numancia y de la que solamente se conocían bronces. El denario ha sido también perforado.
- Fig. 2, c) Ag. 3’61 g , eje 9. Misc lám. $46 \mathrm{n}^{\circ}$ 13; G-B \& B 83, n 28; CNH 233, 13.

- Denarios de ikalesken : según la información de J. Cabré aparecieron en el T. Salvacañete «ocho ejemplares variados y con taladro tres de ellos» ${ }^{12}$. Sin embargo, según M. Gómez Moreno fueron diez los denarios de esta ceca, «de varios tipos hasta los más modernos» ${ }^{13}$. Hoy en el MAN sólo se encuentran depositados cinco y sólo dos de ellos están perforados ${ }^{14}$. Si esta última información es correcta

\footnotetext{
${ }^{12}$ Cabré Aguiló, J., cit. (n. 3), 155

13 Misc., 182.

${ }^{14}$ Navascués, J.M., cit. (n. 9), 38 y 57 nos 52 -56; perforados $n^{\text {os }} 52$ y 53 .
} 
sería necesario aún localizar otros cinco denarios de ikalesken, uno de ellos perforado.

En otro trabajo anterior de Gómez Moreno tenemos ilustrados cuatro ejemplares de este tesoro ${ }^{15} \mathrm{y}$ ninguno de ellos está en el MAN, por lo que hemos intentado identificarlos entre los fondos del IVDJ, donde efectivamente se halla uno, el $\mathrm{n}^{\circ} 12$. El Instituto guarda veinticuatro, casi todos procedentes de la colección Gómez Moreno y, entre ellos, hemos comprobado que sólo uno de los denarios fotografiados por Gómez Moreno está hoy allí, el n ${ }^{\circ} 12$, nuestra figura $2 \mathrm{~d}$. A éste, quizás podríamos añadir otro, perforado, cuya impronta aparece además en el álbum de la colección Gómez Moreno ${ }^{16}$. En el supuesto de que estos datos sean correctos, podríamos decir que el IVDJ posee dos.

- Fig. 2, d) Ag. 3’80 g, eje 11. Vives lám. 66, 1; Gómez Moreno, «Notas...», lám. 4 nº 12; CNH 324,1 .

- Fig. 2, e) Ag. 4’07 g , eje 4, perforado y rellenado. Vives 66, 1; CNH 324, 1.

- Denarios de bolśkan: en esta ocasión Cabré recuenta «cincuenta ejemplares, quince taladrados, de varios tipos (Vives lám. $43 \mathrm{n}^{\circ} 2$ y 3)». Pero esos datos no coinciden con los de Gómez Moreno que nos dice que había «cincuenta y tantos» de este taller. Ahora veremos que esta última información, aunque ambigua, resulta más exacta.

En la publicación de Navascués de los fondos depositados en el MAN se recopilan cincuenta y un denarios de bolskan como procedentes de Salvacañete y entre ellos hay diecisiete perforados; sin duda se trata de los quince que pertenecieron al primer lote ingresado en el Museo más los otros dos, también perforados, pertenecientes al segundo lote ${ }^{17}$. Sabemos además por A. Fernández Avilés que sólo se compraron dos de estas piezas aunque en el ofrecimiento formulado por el vendedor figuraban cuatro ${ }^{18}$.

También entre las piezas ingresadas en el Museo de Cuenca figuran otros dos denarios de esta ceca que M. Osuna supone formaron parte del tesoro.

\footnotetext{
${ }^{15}$ Notas... cit. (n. 1), lám. 4 n ${ }^{\circ} 12-14$ y 17.

${ }^{16}$ La perforación de esta moneda ha sido rellenada pero no hemos podido comprobar si en fecha antigua o reciente. El álbum de la colección Gómez Moreno, manuscrito con improntas, se encuentra en la bilbioteca del IVDJ y pudimos manejarlo gracias a D. Diego Angulo, presidente del patronato y a Dña. Balbina Martínez Cabiró, directora del instituto. Vaya aquí nuestro agradecimiento a ambos por las facilidades dadas para la elaboración del catálogo de las monedas antiguas del Instituto.

${ }^{17}$ Fernández Avilés, A.,cit. (n. 7), 35-38

${ }^{18}$ Ibid., 37 n. 5.
}

Sin embargo, tanto Cabré como Gómez Moreno coinciden en el dato de que había «dos ejemplares forrados, con cabeza pequeña», piezas no localizadas hasta el momento, que no constan entre los fondos del MAN. En el Instituto hemos comprobado la existencia de dos denarios forrados y de un alma de denario, cuyas improntas aparecen en el álbum de don Manuel, y que podrían haber pertenecido al tesoro de Salvacañete.

- Fig. 2, f) Ae 3'12 g, eje 1. Vives 43, 2; CNH 211,6 . Denario forrado.

- Fig. 2, g ) Ae 3’25 g, eje 12. Vives 43, 3; $\mathrm{CNH} 211,6$. Denario forrado.

\section{Piezas no localizadas EN EL IVDJ}

- Denario de iltiŕtaśalirban: también taladrado y, según la catalogación de Gómez Moreno, correspondiente a Vives lám. $26 n^{\circ} 2$. Hay en el Instituto dos piezas de este tipo pero ninguna de ellas presenta perforación alguna y además hemos podido constatar que pertenecieron una a la colección Buckler y la otra a la Jordana, por lo que su entrada en el Instituto es anterior a 1925. Todo ello descarta su posible pertenencia al tesoro de Salvacañete.

- Denario de kese: de nuevo se trata de una moneda taladrada y en este caso ha sido catalogada por Gómez Moreno como Vives lám. $31 n^{\circ} 11$. Esta es una de las piezas que no fueron depositadas en el MAN. En el Instituto existen tres denarios de esta serie, uno de la antigua colección Ferrant, otro de la colección López Soto y el último de la Gómez Moreno, pero ninguno de ellos está perforado. Sin embargo, hace unos años M. Osuna dió a conocer un nuevo lote de joyas y monedas que, al parecer, pudieron haber formado parte inicialmente del tesoro de Salvacañete y que fueron depositadas en una fechas posterior en el Museo de Cuenca ${ }^{19}$; entre ellas hay un denario de kese, perforado, que responde a las siguientes características :

Ag. 4'10 g, eje - . Vives 31,11; Vill. Monedes de Tarraco 17; CNH 160, 17.

\section{SOBRE LA CRONOLOGÍA DE LAS SERIES ROMANO-REPUBLICANAS}

Casi todos los autores coinciden en el dato de que los denarios romano-republicanos hallados en Salva-

\footnotetext{
${ }^{19}$ Osuna Ruiz, M., cit. (n. 10), 390.
} 
cañete eran doce, a excepción de K. Raddatz que habla de once aunque de su recuento resultan doce ejemplares ${ }^{20}$. Estas monedas fueron depositadas en el MAN en 1941 y dadas a conocer por J.M. de Navascués ${ }^{21}$; sin embargo, posteriormente, M. Osuna añade a ese conjunto inicial un nuevo ejemplar, también perforado, depositado en el Museo de Cuenca ${ }^{22}$. En el supuesto de aceptar que este último denario también hubiera formado parte del tesoro de Salvaca- ñete nos encontraríamos que son trece ejemplares romano-republicanos y no doce, pero sobre todo que el nuevo ejemplar del M. de Cuenca es el más antiguo de todos los conocidos del Tesoro hasta el momento.

Puesto que la bibliografía utilizada en el trabajo de Navascués para catalogar estas series está hoy superada, hemos reclasificado este material, siguiendo a M.H. Crawford (RRC), y el resultado es el siguiente:

\begin{tabular}{|r|l|l|l|r|}
\hline $\mathbf{N}^{\mathbf{1}}$ & \multicolumn{1}{|c|}{ Marcas ó Magistrados } & \multicolumn{1}{|c|}{ Ceca } & Cronología & RRC \\
\hline 1 & anónimo & Roma & post. 211 a.C. & $53 / 2$ \\
2 & símbolo punta de lanza & SE. de Italia & $211-210$ a.C. & $83 / 2$ \\
3 & símbolo punta de lanza & SE. de Italia & $211-210$ a.C & $83 / 2$ \\
4 & símbolo tridente & Roma & $206-195$ a.C & $115 / 1$ \\
5 & P. ME & Roma & $194-190$ a.C. & $138 / 1$ \\
6 & anónimo & Roma & $179-170$ a.C & $167 / 1$ \\
7 & NAT & Roma & 149 a.C. & $200 / 1$ \\
8 & NATTA & Roma & 148 a.C. & $216 / 1$ \\
9 & L. SEMP. & Roma & 147 a.C. & $218 / 1$ \\
10 & L. CVP. & Roma & 138 a.C. & $232 / 1$ \\
11 & CN. GEL. & Roma & 100 a.C. & $328 / 1$ \\
12 & P. SERVILI M.F. & Roma & a.C. & $328 / 1$ \\
13 & P. SERVILI M.F. & Roma & \\
\hline
\end{tabular}

Fig. 3.- Nuevas catalogación y cronología de los denarios romano-republicanos del tesoro de Salvacañete.

Es decir, se trata de un conjunto de trece denarios fechados entre el año 211 y el 100 a.C. Pero al examinar de forma minuciosa la distribución cronológica de estas series hemos podido observar que la mitad del conjunto es anterior al 170 a.C. y que la mayoría de las piezas restantes se sitúa en las dos décadas centrales del s. II a.C. El tesoro se cierra con dos denarios del año 100 a.C.; sin embargo, existe un vacío absoluto entre el 138 y el 100 a.C. Este dato resulta anómalo puesto que precisamente en esos años la producción de la ceca de Roma aumenta de forma espectacular ${ }^{23} \mathrm{y}$ en todos los tesoros de la zona meridional peninsular se acrecienta el índice de alimentación monetaria, especialmente en las décadas $139-130$ y $119-110$ a.C. ${ }^{24}$. Lo mismo sucede en el resto de las ocultaciones hispanas llevadas a cabo en el cambio del s. II al s. I a.C. Sólo hemos podido constatar dos excepciones: el tesoro de Chiclana de Segura (Jaén) donde también se observa que faltan ejemplares correspondientes al pe-

\footnotetext{
${ }^{20}$ Raddatz, K., cit. (n. 8), 248-249.

${ }^{21}$ Navascués, J.M., cit. (n. 9), 57-58 n 57-68

${ }^{22}$ Osuna Ruiz, M., cit. (n. 10), 391.

${ }^{23}$ RRC 636 y 699.

${ }^{24}$ No insistiremos en este punto puesto que recientemente ha sido analizado por F. Chaves Tristán en Los Tesoros en el Sur de Hispania. Conjuntos de denarios y objetos de plata durante los s. II y I a.C., Sevilla, 1996, 555-557. El fenómeno se repite en los tesoros de las regiones levantina, meseteña y andaluza.
}

ríodo 123-114 a.C. ${ }^{25}$. y el de Albánchez (Úbeda, Jaén) donde hay un vacío entre el 131 y 121 a.C. y de ahí al cierre la mayoría de los denarios son del $116 / 114$ a.C. ${ }^{26}$.

\begin{tabular}{|c|c|c|c|c|}
\hline & \multicolumn{3}{|c|}{ Piezas conservadas } & \multirow{2}{*}{$\begin{array}{l}\text { Piezas no } \\
\text { localizadas }\end{array}$} \\
\hline & MAN & IVDJ & M. Cuenca & \\
\hline arse & 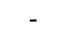 & 2 & - & ninguna \\
\hline ikalesken & 5 & 2 & - & 3 \\
\hline iltiŕtaśalirban & - & - & - & 1 \\
\hline bolśkan & 51 & 2 & 2 & ninguna \\
\hline kese & - & - & 1 & ninguna \\
\hline śekaisa & - & 1 & - & ninguna \\
\hline romano-repub. & 12 & - & 1 & ninguna \\
\hline TOTAL & 68 & 7 & 4 & 4 \\
\hline
\end{tabular}

Fig. 4.-Intento de reconstrucción del tesoro de Salvacañete (Cuenca).

En resumen, podemos afirmar que el número de monedas aparecidas en Salvacañete, y actualmente depositadas en distintas instituciones, es superior a

${ }^{25}$ Avellá, L. y Rodríguez, P., Un tesoro de plata procedente de Chiclana de Segura (Jaén), BIEG 126, 1983, 23-41. Este conjunto se componía de 38 piezas de orfebrería y joyas, material de fundición y un total de monedas desconocido del que se han estudiado 22 denarios romano-republicanos fechados entre 211 y 114 a.C.

${ }^{26}$ Pérez Sindreu, F. , Tesorillo de Albánchez (Jaén), GN 74-75, 1984, 107-111. Composición: 16 denarios romanorepublicanos fechados entre 211 y 106 a.C. 
la cifra recogida inicialmente por J. Cabré. Se sabe que el conjunto permaneció durante algún tiempo en el mercado de antigüedades de Madrid, antes de ser adquirido oficialmente por el Estado español, y por ello es posible que los coleccionistas, o bien simples familiares, compraran algunas de las piezas, aunque no haya quedado constancia de ello por escrito ${ }^{27}$. El tesoro de Salvacañete presenta una distribución temporal muy desigual y exenta de paralelos que no se puede relacionar con el volumen de emisiones de la ceca de Roma y que quizás esté vinculada al posible carácter votivo del ocultamiento. Veámoslo.

\section{COMENTARIO HISTÓRICO}

Salvacañete está en la confluencia del río Cabriel y el arroyo de las Salinas, en la vertiente oriental de la sierra de El Escornadero, lugar apropiado para un culto de una divinidad en relación con los bosques $\mathrm{y}$ con las aguas y por lo tanto curativa, a la que posiblemente iría dedicada la laminita con representación de ojos del tesoro, pensada para ser pendida por los dos orificios superiores laterales ${ }^{28}$. También podrían ser ojos la decoración en «ochos» con glóbulo central de las arracadas de los pendientes ${ }^{29}$. En los tres ejemplares se repiten los ochos y las puntas de lanza; otra punta de lanza aparece además suelta en el tesoro, como posible exvoto, desde luego no utilitario dado su pequeño tamaño y el que sea de plata. Estas puntas de lanza pueden estar en relación con la caza, en el culto de una divinidad, como veremos, referida constantemente a animales, e incluso haber sido homologada con Artemis-Diana. Más aún, los dos objetos similares entre sí, en forma de cartucho colgante aparecidos en el tesoro como exvotos, podrían estar miniaturizando los carcajs propios de la divinidad, quizás incluso copia exacta puesto que son homólogos entre sí ${ }^{30}$.

Pasemos ahora al objeto más descriptivo para nosotros (fig. 5). Es la plaquita decorada en repujado por una cara frontal humana, un ave y una abeja y preparada también para ser colgada por el orificio superior ${ }^{31}$. La placa ha sido decorada con abundantes globulitos, huevos repujados que rodean la cara, la abeja y en parte el ave; ésta, incluso toma con su

\footnotetext{
${ }^{27}$ Este podría muy bien ser el caso de las monedas que pertenecieron a la colección de don Manuel Gómez Moreno, que hoy conocemos gracias al álbum de improntas, y el del lote donado en una fecha más tardía al Museo de Cuenca.

${ }^{28}$ Raddatz, cit. (n. 8), lám.53,30.

${ }^{29}$ Ibm., lám. 53, 25-27.

${ }^{30} \mathrm{Ibm}$., lám. 52, 14, 15.

${ }^{31} \mathrm{Ibm}$, lám. 50, 5.
}

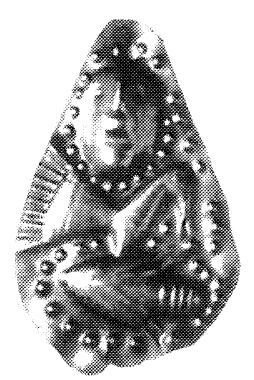

Fig. 5.-Plaquita de plata del Tesoro de Salvacañete. MAN 37-093. Esc. 1:1

pico uno de esos globulitos dándole así una simbología que excede la simplemente decorativa. No se puede discernir el tipo de ave, una paloma, una grulla por su largo pico, no sabemos. Además acompaña a todo ello una abeja.

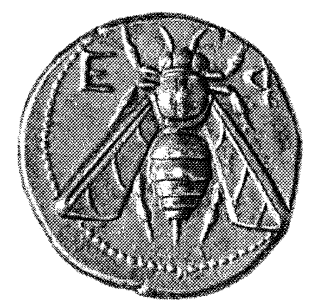

Fig. 6.-Tetradracma de Ephesos de 370-340 aC. 15'31 g, 24 mm, BM.1929.

La abeja es un insecto rara vez utilizado y por lo tanto más determinante. En el munco clásico, el único del que tenemos suficiente documentación para estudiarla, su comportamiento es frecuentemente homologado con el humano por su vida en sociedad, por su jerarquía, reproducción, habilidad y riqueza industrial, a la vez de por su capacidad de defensa y agresividad cuando es necesario. Por esta simbología es animal sagrado de Artemis Efesia, más en relación con la divinidad anatólica que con la griega, a cuya vera aparece frecuentemente ilustrada e, incluso, es la abeja la que representa a $\mathrm{Ar}$ temis, ella sola, en las monedas de Efeso (fig. 6) ${ }^{32}$. En Ampurias aparece ilustrada como símbolo en las dracmas, y aunque es conocida la dificultad que conlleva la identificación de la divinidad emporitana efigiada como Aretusa o Perséfone, parece hoy aceptarse que en realidad estamos ante la imagen de Artemis, que la ceca en monedas latinas más tar-

${ }^{32}$ C. Kraay: Archaic and Classical Greek Coins, Berkeley \& Los Angeles, 1976, 256. lám. 53, 904. 
días efigiará con carcaj y arco, claramente como Diana ${ }^{33}$.

Las monedas proporcionan además datos complementarios que en parte hemos comentado arriba. La ausencia de ciertos denarios fechados entre los años 138 y 100 a.C. es digna de atención. El análisis de las piezas conservadas parece indicar que el atesoramiento siguió un criterio selectivo. El tesoro contiene una muestra representativa de todas las emisiones denariales romano-republicanas hasta el año 100 en que se cierra, sólo mientras las imágenes del reverso representen caballos - Dioscuros, bigas o cuadrigas-, excluyéndose todas las emisiones con iconografía «familiar» que a partir de año 137 a.C. es habitual en los reversos de los denarios romanos en que los triunviri monetales sacan a la luz sus historias míticas ${ }^{34}$. Pues bién, no hay ningún denario de este tipo «familiar» y sólo aquellos que ilustran caballos, lo mismo que ocurre con las monedas hispánicas, denarios de jinete y dracmas con toro de Sagunto, excluyéndose por ejemplo las dracmas emporitanas o de imitación - con Pegaso, animal irreal- que sabemos siguen atesorándose en abundancia hasta época post-sertoriana, es decir mucho más tarde que el cierre del tesoro de Salvacañete, donde, por tanto, podrían haber sido recogidas ${ }^{35}$.

Otro detalle más llama la atención: el cuidado puesto a la hora de taladrar todas estas monedas para que en ningún caso la perforación afecte de forma significativa a los animales de los reversos, sino que el agujero se sitúe delante o detrás de la cabeza de los jinetes, nunca en las partes vitales. Todo ello nos hace sospechar que la iconografía de la moneda pudo ser un elemento decisivo a la hora de conformar el tesoro, e incluso pudo haber actuado como un código para seleccionar las piezas de esta ocultación, para la que ya desde un primer momento J. Cabré eligió el calificativo de depósito vo-

${ }^{33}$ CNH 28,79; Pena, M.J.: Artemis-Diana y algunas cuestiones en relación con su iconografía $\mathrm{y}$ su culto en Occidente, Ampurias 35, 1973;

${ }^{34}$ RRC emisiones $n^{\circ} 234$ a 286; 137-115 a.C.

${ }^{35}$ V.g. el tesoro de Aoristá, cf. García-Bellido, M.P.: Las relaciones económicas entre Massalia, Emporion y Gades a través de la moneda, Huelva Arqueológica XIII, 2, 131. tivo $^{36}$. Ahora, la magnitud del tesoro, la longitud en el tiempo de las monedas y la presencia de laminitas, pendientes y otros objetos con iconografía que podríamos interpretar como religiosa, parece indicar que efectivamente, como J. Cabré supuso, estamos ante el depósito votivo de un santuario, aunque quizás nunca sabremos si procedía de una favissa.

Hemos hablado de Artemis, pero no es fácil sin embargo que el santuario, si es que existió, estuviese dedicado a Artemis sino más posiblemente a una divinidad local femenina por los símbolos de fertilidad de los objetos, pero también una potnia theron a juzgar por los caballos y toros, dos conceptos que podrían parecer antagónicos y que sin embargo están atestiguados conjuntamente en muchas divinidades femeninas, como por ejemplo Astarté.

En la misma provincia de Cuenca, en Segóbriga, tenemos bien atestiguado un santuario a Diana conocido desde antiguo y que ha merecido mucha atención ${ }^{37}$. En las inscripciones en roca Diana es llamada Domina y Frugifera, epítetos que a G. Alföldy le han merecido el juicio de que la divinidad tuviera una esencia de diosa de la naturaleza, de la vegetación, de la fertilidad más que de la caza, explicando que la mayoría de sus devotos fuesen mujeres, como las incripciones indican ${ }^{38}$.

Naturalmente nada sabemos sobre el posible santuario de Salvacañete, y lo que aquí hemos presentado como interpretación de los objetos son sugerencias que habrán de confrontarse con el resto del tesoro y con el contexto arqueológico, si es que puede reconstruirse, pero el paralelo del santuario de Segóbriga apoyaría la presencia, en esa serranía de Cuenca, de una divinidad que los griegos y romanos identificarían con Artemis y Diana. Sin embargo, sí es una contribución segura al acopio de las piezas que faltaban por estudiar, aquéllas guardadas en el Instituto Valencia de Don Juan.

${ }^{36}$ Cabré Aguiló, J., cit. (n. 2), 151. Para otros tipos de selecciones de monedas con fines cultuales cf. Olmos, R. : Usos y lecturas de la moneda en la Hispania prerromana, en M.P. García-Bellido \& R. M. Centeno (edts) La Moneda hispánica: ciudad y territorio, Anejos de AEspA, XIV, Madrid 1995, 43.

${ }^{37} \mathrm{Cf}$. por último, Almagro-Gorbea, M.: El lucus Dianae con inscripciones rupestres de Segobriga, en Rodríguez Colmenero, A., Saxa Scripta, (inscripciones en roca), Anejos de Larouco 2, A Coruña 1995, 61-97

${ }^{38}$ Alföldy, G.: Epigraphica Hispanica VI. Das Diana-Heiligtum von Segobriga, ZPE 58, 156-159 